International Journal of Pure and Applied Mathematics

Volume 98 No. 2 2015, 239-259

ISSN: 1311-8080 (printed version); ISSN: 1314-3395 (on-line version)

url: http://www.ijpam.eu

doi: http://dx.doi.org/10.12732/ijpam.v98i2.7

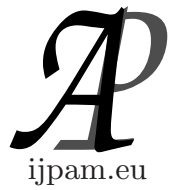

\title{
BOOLEAN ALGEBRAS AND CLASSIFICATION OF INTERACTIONS IN SUFFICIENT-COMPONENT CAUSE MODEL
}

\author{
Vladimir G. Panov ${ }^{1}$ §, Julia V. Nagrebetskaya ${ }^{2}$ \\ ${ }^{1}$ Institute of Industrial Ecology of Ural Branch of RAS \\ S. Kovalevskaya st., 20, Ekaterinburg, RUSSIA \\ ${ }^{2}$ Ural Federal University \\ V. Lenin pr., 51, Ekaterinburg, RUSSIA
}

\begin{abstract}
A mathematical model of the sufficient-component cause framework is considered based on the theories of Boolean algebra. The model consists of the space of states of a binary experiment and a set of symmetries of the experiment. The space of states is a Boolean algebra of $n$ Boolean variables where $n$ is the number of the binary causes in the experiment. The set of symmetries of the experiment is a subgroup of the group of all automorphisms of Boolean algebra of the states of experiment. This subgroup is generated by transformations preserving a type of interaction. An experimenter should deduce these transformations from the peculiar properties of the experiment. Examples of such transformations are provided. Classification of interactions is obtained by the calculation of the orbits of action of the group of symmetries on the space of states of the experiment. It is shown that the classification of the interaction for the ordinary symmetries of sufficient causes is the same as reported in related works. Other symmetries of the binary experiment are considered as well. It is shown that the corresponding classification of the interaction types in a binary experiment depends substantially on the symmetries of the experiment. Statistical criteria of particular types of responses are proven and the problem
\end{abstract}

Received: October 1, 2014

(C) 2015 Academic Publications, Ltd.

${ }^{\S}$ Correspondence author url: www.acadpubl.eu 
of mutual antagonism is discussed in the Appendix.

AMS Subject Classification: 03G05, 06E30, 94C10, 22F50

Key Words: sufficient component cause model, Boolean automorphisms, group action, orbits, synergism, antagonism

\section{Introduction}

Causality is the central aim of many studies in various sciences. However, there are substantial difficulties in the formalization of the concepts of cause and consequence, which have their specific features in every particular science. Nevertheless, the common properties of these notions, which may be formulated as a philosophical conceptualization, allow us to investigate important causality properties. Some of these properties might have had a formal representation and application in particular of these properties might have had a formal representation and application in particular sciences. Thus, following mathematical and epidemiological reasoning will be based on Mackie's philosophical analysis of causality [16] and [17]. Mackie clarified formulation, the problem of causality, and suggested possible research methods. In particular, he widely used formallogical relations arising among events, which have created a sufficient cause. Though Mackie was mainly interested in the general properties of causality related to a single event (singular causation), his researches were soon applied in epidemiology [18] and [28].

Particularly important is the article of Rothman [28] who presented a readable and practical application of the Mackie's methodology in epidemiology. Essential extension of Mackie's method was the consideration of several causal mechanisms leading to the outcome of interest (e.g. disease or death). Every causal mechanism is represented as a set of simultaneously presented events, i.e., the conjunction of the events. We may conceive causality as the outcome of the set of all its causal mechanisms, such that, if we were to list all sufficient causes, then, their disjunction would be equivalent to the outcome. This approach to causality investigation was named sufficient-component cause framework in epidemiology. Firstly, it was mainly applied for the illustration of relationships among different causal mechanisms and their parts for the given disease. It has made the understanding of causality in epidemiology deeper. For instance, Rothman [28] paid much attention to the clarification of the distinction between effect modification and confounding, and his graphical presentation of causal mechanisms as "causal pies"[19] and [28] clarifies that the sum of attributable fractions need not equal 1 because of the joint presence of multiple causes in 
the same sufficient cause.

The sufficient causes model [28] enabled a clearer analysis of the synergism of sufficient causes. However, rigorous results were mostly obtained in the binary case (two factors and the outcome took two values). We present below that the correct statement of the problem of classification of interaction in the general case requires specific algebraic notions.

The first formal presentation of the sufficient causes theory (also for the binary case) was introduced by Miettinen [20]. Two factors, $X_{1}$ and $X_{2}$, and the outcome $D$, presented by Miettinen, took on 2 values, and he had shown how to reduce the sufficient-cause framework to counterfactual framework in this case.

This allowed sufficient causes to be presented within the Neyman-HollandRubin causality theory [14], [21], [26]. Let us denote the reference categories of the factors $X_{1}$ and $X_{2}$ with 0 and $1, D_{x_{1} x_{2}}$ is denoted as the value of outcome $D$ under the condition $X_{1}=x_{1}, X_{2}=x_{2}, \quad x_{i}=0,1, i=1,2 . D_{x_{1} x_{2}}$ determines a real or potential response of an individual on the exposure of factors at the given combination of their levels. A response type for the given individual is the set of values $\left(D_{00}, D_{10}, D_{01}, D_{11}\right)$ (below it is named as full response). Miettinen [20] argued for a classification of the factor's interaction types based on the kind of full response. Furthermore, he suggested a term for every type of full response, which reflects the character of the interaction or the absence of the interaction. Unfortunately, there were logical drawbacks in Miettinen's classification that were noticed by Greenland and Poole [10].

Recoding or changes in reference levels are often free, for instance, as it is for GENDER [10]. As it is said in [10] "without the adoption of a deeper theory about how effects result, no reference category can be regarded as correct" and "it would seem worthwhile to discover what, if any, properties of joint action are invariant under recoding or changes in reference levels." Greenland and Poole [10] analyzed the Miettinen's classification [20] and found that the required invariance was not holding. They suggested refining the classification to take into account the invariance under change in the reference categories. To do this they defined seven response classes, which are invariant under recoding. Thus, one should interpret the properties of joint action of the whole class rather than a particular response. That is the responses composed of an invariant class have the same type of joint action of the given factors. Later it another natural invariant property was noticed in [33], i.e., the transposition of factors. Thus, the seven invariant classes of [10] were reduced to six by joining two classes together [33].

Generally, one can say that all possible full responses in the binary experi- 
ment are divided into disjoint classes, which have the same joint action properties. This partition is determined by the natural symmetries of the experiment, for instance, swapping of the factors or recoding their levels. These principles of the binary experiment were declared in [10] and [33] in implicit form, though their dissemination to the general case was difficult owing to the lack of the appropriate mathematical formalization of the sufficient causes model. Thus, for example, there are the general formal schemes [1], [34], [36] of the sufficient causes, but the classification of joint action was not considered. It is unlikely that the existing classification of the joint action [10] and [33] can be extended to many binary factors or to multilevel factors.

Despite the relatively simple formalization of the initial statements of sufficient causes, the theory rapidly develops, and researchers have discovered fruitful relationships with the various branches of mathematics. Numerous applications of abstract algebra in sufficient causes were demonstrated in [1] and [34]. Statistical methods were used in sufficient causes long ago [2], [15], [20], [32], [33], and this trend continues to grow [29], [31], [35], [37]. The position of the sufficient-component cause model among other causal modelling methods presented in [9].

The following issues are considered below. A mathematical framework for sufficient causes will be suggested for an arbitrary number of binary factors. The framework takes into account the pattern of full response and those symmetries that are presented in the experiment. Specifically, the binary sufficient-causes model will be reduced to the theory of (finite) Boolean algebras. To avoid mathematical details, we only consider the binary case. Some remarks concerning the general case are given in the text. The general construction for an arbitrary number of binary factors will be obvious thereafter. The Boolean algebra of the full responses is called Boolean algebra states of experiment.

To classify the joint action in sufficient causes one needs to know symmetries of the experiment that were used in [10] and [33] in the implicit form. In the proposed formalization, these symmetries are represented as automorphisms on the Boolean algebra states of experiment. The automorphisms generate a group of automorphisms by acting on the Boolean algebra states of experiment. The group is a subgroup of the group of all automorphisms of the Boolean algebra. Thus, response classes expressing the same type of the joint action turns out to be orbits of action of the automorphism group on the Boolean algebra states of the experiment.

The algebraic formalism considered in the article has been proposed in an initial form in [24]. The algebraic issues for the sufficient causes symmetries presented in detail in [25]. Statistical criteria for particular responses consid- 
ered in Appendix were introduced in [22]. A method for the calculation of an estimate of probability of a particular response was suggested in [23].

The obtained classification is compared to the known classification [10] and [33]. In particular, the notion of synergism/antagonism is discussed given the new classification of joint action.

\section{Space of States of the Binary Experiment}

Binary experiment is considered as such an experiment, in which the response and two acting factors take on values 0 or 1 . More generally, Boolean experiment is defined as such an experiment with binary response, in which $n$ Boolean factors are involved. In order to obtain a mathematical representation of the binary or Boolean experiment, it is convenient to introduce the space of all full responses and the set of admissible transformations, which do not change a type of the joint action. The first space is called the space of states of experiment, and the second the set of symmetries of experiment. Now we consider the mathematical representation of each of them.

Suppose that $D$ and two of its causes, $X_{1}$ and $X_{2}$, are binary variables taking the values 0 or 1 . According to [1] we use the term 'factor' for $X_{i}$, along with 'causes'. It is not obligatory that the value 0 means the absence of the factor, and 1 its presence in the studied measure (or dose). The response denotes $D$, and its levels also are 0 or 1 . In contrast to factors $X_{1}$ and $X_{2}$, the levels of the response $D$ are not generally interchangeable. For definiteness, the event of interest is denoted $D=1$ (below we write shortly $D$ ). To formalize the notion of a cause one could use the notion of nontrivial implication (adapted from the more general discussion in [1])

Definition 1. The Boolean variable $X$, defined on a set $\Omega$, is called an nontrivial implication cause of the response $D$, if $\forall \omega \in \Omega \quad(X(\omega)=1) \Rightarrow$ $(D(\omega)=1)$.

The fact that factors $X_{1}$ and $X_{2}$ are the causes for $D$ means that each of them is a nontrivial implication for $D$, i.e., $X_{1}$ and $X_{2}$ are nontrivial (both are not trivially 0 ) and $X_{i}(\omega)=1 \Rightarrow D(\omega)=1$ for any individual $\omega$. Instead of $X_{i}(\omega)=1$, herein it may be $\bar{X}_{i}(\omega)=1$, as the reference categories of factors are chosen at will. One can easily deduce that if $X_{1}$ and $X_{2}$ are the causes for $D$, then $D$ is a Boolean function of variables $X_{1}$ and $X_{2}$ (for the terminology see [6] and [8]).

There are certain correspondences between the response types and sets of sufficient causes [10]. Greenland and Poole enumerate nine different sufficient 
causes, each involving some combination of $X_{1}$, and $X_{2}$ and their complements along with certain binary background causes $A_{0}, A_{1}, A_{2}, A_{3}, A_{4}, A_{5}, A_{6}, A_{7}$, and $A_{8}$. The nine different sufficient causes that Greenland and Poole gave are then $A_{0}, A_{1} X_{1}, A_{2} \bar{X}_{1}, A_{3} X_{2}, A_{4} \bar{X}_{2}, A_{5} X_{1} X_{2}, A_{6} \bar{X}_{1} X_{2}, A_{7} X_{1} \bar{X}_{2}$ and $A_{8} \bar{X}_{1} \bar{X}_{2}$. Given the nine background causes, we thus have that ( $\vee$ denotes disjunction of events and bar over symbol denotes the complement event)

$$
\begin{array}{r}
D=A_{0} \vee A_{1} X_{1} \vee A_{2} \bar{X}_{1} \vee A_{3} X_{2} \vee A_{4} \bar{X}_{2} \vee A_{5} X_{1} X_{2} \vee \\
A_{6} \bar{X}_{1} X_{2} \vee A_{7} X_{1} \bar{X}_{2} \vee A_{8} \bar{X}_{1} \bar{X}_{2}
\end{array}
$$

Thus, in (1) we see the possible ways by which the event $D$ may occur in the dependence of the factors' values. The Boolean variables $A_{i}$ depend on the individual, and do implement a particular way by which results in $D=1$. More formally, (1) represents the Boolean function $D$ as a disjunction of conjunctions of the Boolean variables $X_{1}$ and $X_{2}$. This representation is convenient to understand the dependence of the response $D$ from the variables $X_{1}$ and $X_{2}$, but generally, it is redundant and inconvenient for further analysis.

The problems discussed below are to determine which full responses express the same type of the combined action of the factors. Thus, different representations of the response $D$ as Boolean function of the variables $X_{1}$ and $X_{2}$ may be used. We use the perfect disjunctive normal form ([6] and [8]) for the representation of the response $D$. Let us denote $D_{i j}$, the value of the response $D$ under conditions $X_{1}=i$ and $X_{2}=j, i, j=0$ or 1 . Then

$$
D=D_{00} \bar{X}_{1} \bar{X}_{2} \vee D_{01} \bar{X}_{1} X_{2} \vee D_{10} X_{1} \bar{X}_{2} \vee D_{11} X_{1} X_{2}
$$

Here, as usual, the conjunction sign is omitted. The uniqueness of the representation (2) for arbitrary Boolean function is proven in the courses of mathematical logic and Boolean functions (see e.g. [6] and [8]).

Definition 2. The space of states of binary experiment is the Boolean algebra $\mathbf{B}\left(X 1, X_{2}\right)$ of Boolean functions of two Boolean variables $X_{1}$ and $X_{2}$.

In general, the space of states of experiment with $n$ binary factors $X_{1}, X_{2}$, $\ldots, X_{n}$ is the Boolean algebra $\mathbf{B}\left(X_{1}, X_{2}, \ldots, X_{n}\right)$ of Boolean functions depending on $n$ Boolean variables. The space of states of experiment with $n$ binary factors consists of $2^{2^{\mathrm{n}}}$ elements. In particular, the space of states of the binary experiment (having two binary factors and a binary response) has 16 elements [10], [20], [33]. 


\section{Symmetries of the Binary Experiment}

The transformations that preserve a type of combined action in the experiment form a substantial part of the modeling of the binary experiment. It is worth recognizing here that we do not discuss the question 'what is the combined action?' or 'what is the interaction?' Instead, we assume that an experimenter clearly understands that some transformations in his or her experiment do not change a character of the combined action of factors. For instance, it is obvious that the type of combined action of factors $X_{1}$ and $X_{2}$ and factors $X_{2}$ and $X_{1}$ is the same. Formally, this means that the type of combined action is not affected by the transposition factors.

From this viewpoint, no transformations were considered by Miettinen in [20], so any full response $\left(D_{00}, D_{10}, D_{01}, D_{11}\right)$, i.e. a Boolean function, represents a particular type of the combined action. Taking into account invariance under transposition, we obtain 12 classes of the combined action.

On the other hand, the invariance under recoding of the reference categories was considered in detail [10], and taking account of this symmetry resulted in seven response classes. These classes may be presented by the following equalities (used notations [10])

$$
\begin{aligned}
& C_{D}=\{1\}, C_{I}=\{0\}, C_{X_{1}}=\left\{X_{1}, \bar{X}_{1}\right\}, C_{X_{2}}=\left\{X_{2}, \bar{X}_{2}\right\}, \\
& C_{M}=\left\{X_{1} X_{2} \vee \bar{X}_{1} \bar{X}_{2}, \bar{X}_{1} X_{2} \vee X_{1} \bar{X}_{2}\right\}, \\
& C_{S}=\left\{X_{1} X_{2}, \bar{X}_{1} X_{2}, X_{1} \bar{X}_{2}, \bar{X}_{1} \bar{X}_{2}\right\}, \\
& C_{T}=\left\{X_{1} \vee X_{2}, \bar{X}_{1} \vee X_{2}, X_{1} \vee \bar{X}_{2}, \bar{X}_{1} \vee \bar{X}_{2}\right\}
\end{aligned}
$$

The invariance relative transposition merges classes $C_{X_{1}}$ and $C_{X_{2}}$ that gives the classification [33]. It is worth mentioning that the logical expressions in every class represent the same type of combined action, though they are not equal. Classes $C_{M}$ and $C_{S}$ are usually mentioned as classes that represent the synergism or antagonism in the binary experiment ([10] and [33]). As for class $C_{S}$ it is the common stand to go back to Mackie [16], [17] and Rothman [28]. A more complicated structure of the responses in $C_{M}$ requires additional argumentation to be considered a synergistic response. In the Appendix, this question is discussed in more detail. There are good reasons for not considering these responses to be neither synergistic nor antagonistic.

The ideas concerning invariant transformations may be formalized within the discussed model of the binary experiment as follows. Transposition of factors is such a transformation $T_{1}$ that acts on the independent variables $X_{1}$ and 
$X_{2}$ by the rule

$$
\begin{array}{ll}
T_{1}\left(X_{1}\right)=X_{2}, & T_{1}\left(X_{2}\right)=X_{1} \\
T_{1}\left(\bar{X}_{1}\right)=\bar{X}_{2} & T_{1}\left(\bar{X}_{2}\right)=\bar{X}_{1}
\end{array}
$$

Similarly, invariance under reference categories recoding [10] may be presented as the transformation $T_{2}$ acting by the rule

$$
\begin{array}{ll}
T_{2}\left(X_{1}\right)=\bar{X}_{1} & T_{2}\left(X_{2}\right)=X_{2} \\
T_{2}\left(\bar{X}_{1}\right)=X_{1} & T_{2}\left(\bar{X}_{2}\right)=\bar{X}_{2}
\end{array}
$$

Those requirements that are given in the implicit form in [10], [31] and [33] required that an interaction type of the operating factors should be invariant under these transformations. This condition is not a formal mathematical one; rather it is empirically motivated by peculiar properties of the experiment. In one experiment, the combined action would be invariant under some transformations, and in another experiment under another. The considered mathematical model shows how to obtain the consequences from one or the other assumptions about the existing symmetries.

The introduced transformations $T_{1}$ and $T_{2}$ act on the independent variables $X_{1}$ and $X_{2}$. Since $\mathbf{B}\left(X_{1}, X_{2}\right)$ is the free Boolean algebra ([6] and [8]), these transformations may be extended on the whole $\mathbf{B}\left(X_{1}, X_{2}\right)$ as follows:

$$
\begin{aligned}
& T_{i}\left(f\left(X_{1}, X_{2}\right)\right)= \\
& f_{00} T_{i}\left(\bar{X}_{1} \bar{X}_{2}\right) \vee f_{01} T_{i}\left(\bar{X}_{1} X_{2}\right) \vee f_{10} T_{i}\left(X_{1} \bar{X}_{2}\right) \vee f_{11} T_{i}\left(X_{1} X_{2}\right),
\end{aligned}
$$

where

$$
\begin{aligned}
& T_{1}\left(X_{1}^{\alpha} X_{2}^{\beta}\right)=X_{1}^{\beta} X_{2}^{\alpha}, T_{2}\left(X_{1}^{\alpha} X_{2}^{\beta}\right)=X_{1}^{\bar{\alpha}} X_{2}^{\beta}, \\
& \alpha, \beta=0,1, \bar{\alpha}=1-\alpha, X_{i}^{0}=\bar{X}_{i}, X_{i}^{1}=X_{i} .
\end{aligned}
$$

Then, $T_{1}$ and $T_{2}$ become automorphisms on the Boolean algebra $\mathbf{B}\left(X_{1}, X_{2}\right)$ [13].

For instance, let the combined action of factors $X_{1}$ and $X_{2}$ on the response $D$ be presented by the Boolean function $X_{1} \bar{X}_{2}$, i.e., joint presence of events $X_{1}=1$ and $X_{2}=0$ results in the event $D=1$. Then, $T_{1}$ and $T_{2}$ transform the function $X_{1} \bar{X}_{2}$ as follows:

$$
T_{1}\left(X_{1} \bar{X}_{2}\right)=\bar{X}_{1} X_{2}, T_{2}\left(X_{1} \bar{X}_{2}\right)=\bar{X}_{1} \bar{X}_{2} .
$$

Since transformations $T_{1}$ and $T_{2}$ can be applied successively, the set of symmetries of binary experiment is really a group generated by the automorphisms $T_{1}$ and $T_{2}$ (see, e.g. [7] and [11]). 
Definition 3. A group of Boolean automorphisms defined on the space of states of a Boolean experiment is called the group of symmetries of the experiment.

Thus, the formal model of the binary experiment with two factors consists of the Boolean algebra of two variables (space of states of experiment) and a group of automorphisms that act on the Boolean algebra (group of symmetries of experiment). Similarly, for Boolean experiment, the space of states is the Boolean algebra of $n$ variables, and the set of symmetries is a group of automorphisms acting on the space of states.

\section{Classification of the Joint Action in the Binary Experiment}

The mathematical model of the binary experiment allows obtaining the classification of the joint action of factors by a standard mathematical routine. Since the transformations $T_{1}$ and $T_{2}$ express invariance properties of the type of combined action, those full responses that may be obtained from each other by a sequence of transformations $T_{1}$ and $T_{2}$ (and their inverses) exhibit the same type of joint action of factors $X_{1}$ and $X_{2}$. Mathematically, this means that all the full responses located in the same orbit under the action of the symmetries group ([7] and [11]) represent the same type of the joint action. In other words, classification of combined action of factors is the partition of the space of states of experiment under the action of the group of symmetries of the experiment. This is a well-known mathematical problem that may be treated by various methods [3], [4], [5], [12], [27].

Theorem 4. The symmetry group of the binary experiment, generated by the transformations $T_{1}$ and $T_{2}$ is isomorphic to the dihedral group $D_{8}$.

Proof. Let us denote $G_{1}$ the group generated by T1 and T2. A short way to ensure that $G_{1}$ is the dihedral group $D_{8}$ is the checking of the properties that $G_{1}=\left\langle S_{1}, S_{2} \mid S_{1}^{4}=S_{2}^{2}=e, S_{2} S_{1}=S_{1}^{-1} S_{2}\right\rangle$ for some transformations $S_{1}$ and $S_{2}$ from $G_{1}$ (see, e.g. [7] and [11]). We can take the following transformations: $S_{1}=T_{1} \cdot T_{2}$ and $S_{2}=T_{2}$. Omitting the obvious calculations we finish the proof.

Theorem 5. The orbits of the action of group $D_{8}$ on the Boolean algebra 
$\mathbf{B}\left(X_{1}, X_{2}\right)$ are

$$
\begin{aligned}
& \{0\}, \quad\{1\}, \quad\left\{X_{1}, \bar{X}_{1}, X_{2}, \bar{X}_{2}\right\}, \\
& \left\{X_{1} \vee X_{2}, \bar{X}_{1} \vee X_{2}, X_{1} \vee \bar{X}_{2}, \bar{X}_{1} \vee \bar{X}_{2}\right\}, \\
& \left\{X_{1} X_{2}, \bar{X}_{1} X_{2}, X_{1} \bar{X}_{2}, \bar{X}_{1} \bar{X}_{2}\right\}, \\
& \left\{X_{1} X_{2} \vee \bar{X}_{1} \bar{X}_{2}, \bar{X}_{1} X_{2} \vee X_{1} \bar{X}_{2}\right\}
\end{aligned}
$$

Proof. The proof can be obtained by direct calculations, or using an appropriate mathematical software (GAP, Mathematica and other software).

It is important to notice that the responses $X_{1} X_{2} \vee \bar{X}_{1} \bar{X}_{2}$ and $\bar{X}_{1} X_{2} \vee X_{1} \bar{X}_{2}$ form a separate class, though they are often considered along with conjunctions $X_{1} X_{2}$ (e.g. [31] and [33]).

The Boolean functions in each class represent a particular type of joint action, which describes as follows [10] $\{1\}$, the doomed type; $\{0\}$, the immune type;

$\left\{X_{1}, \bar{X}_{1}, X_{2}, \bar{X}_{2}\right\}$, the type in which only one factor is effective, but the other is not;

$\left\{X_{1} X_{2} \vee \bar{X}_{1} \bar{X}_{2}, \bar{X}_{1} X_{2} \vee X_{1} \bar{X}_{2}\right\}$, the type exhibiting the mutual antagonism; $\left\{X_{1} X_{2}, \bar{X}_{1} X_{2}, X_{1} \bar{X}_{2}, \bar{X}_{1} \bar{X}_{2}\right\}$, the type in which disease occurs for only one exposure combination;

$\left\{X_{1} \vee X_{2}, \bar{X}_{1} \vee X_{2}, X_{1} \vee \bar{X}_{2}, \bar{X}_{1} \vee \bar{X}_{2}\right\}$, the type in which disease occurs for three exposure combinations.

In particular, functions $\left\{X_{1} X_{2} \vee \bar{X}_{1} \bar{X}_{2}, \bar{X}_{1} X_{2} \vee X_{1} \bar{X}_{2}\right\}$ have some different type of combined action than conjunctions

$\left\{X_{1} X_{2}, \bar{X}_{1} X_{2}, X_{1} \bar{X}_{2}, \bar{X}_{1} \bar{X}_{2}\right\}$, though both classes are usually considered as representing synergism or antagonism (see, e.g. [10] and [33]). In the Appendix, we have proved statistical criteria (sufficient conditions) of the existence of an individual with a particular response, including full responses $(1,0,0,1)$ and $(0,1,1,0)$, which are presented by functions $X_{1} X_{2} \vee \bar{X}_{1} \bar{X}_{2}$ and $\bar{X}_{1} X_{2} \vee X_{1} \bar{X}_{2}$ respectively. It is implied that such individuals are presented quite often in a binary experiment, thus, to consider these responses to be synergistic or antagonistic is doubtful.

\section{Other Symmetries of the Binary Experiment}

The suggested mathematical model of the Boolean and binary experiment allows researchers to set up a problem of combined action classification both in a wider context and more correctly. As we saw, the combined action types are determined by the symmetries of the experiment. Transformations $T_{1}$ and $T_{2}$ are 
usually considered in sufficient causes theory (see (3)-(4)), which generates the dihedral group $D_{8}$ as the group of symmetries, and it creates the classification (6).

Nevertheless, experimental conditions may have other allowable transformations that preserve the joint action types. Apparently the transposition $T_{1}$ has to be presented in any classification method. On the other hand, the transformation $T_{2}$ may not be presented in an experiment. Then the group of symmetries of an experiment would be a subgroup of the group of all automorphisms of the Boolean algebra $\mathbf{B}\left(X_{1}, X_{2}\right)$. Since the latter group, namely, the permutation group $S_{4}$, is well known (see e.g. [7] and [11]), the possible groups of symmetries of the binary experiment are those subgroups of $S_{4}$ that include the transformation $T_{1}$. The list of all $S_{4}$ subgroups is presented in related mathematical resources [3], [4], [5], [30]. This allows a researcher to accomplish analysis of the joint action types regarding the presented symmetries.

For instance, let us consider a binary experiment with admissible transformations $T_{1}$ (see (3)) and $T_{3}$

$$
\begin{aligned}
& T_{3}\left(X_{1}\right)=\bar{X}_{1}, T_{3}\left(X_{2}\right)=\bar{X}_{2} \\
& T_{3}\left(\bar{X}_{1}\right)=X_{1} \quad T_{3}\left(\bar{X}_{2}\right)=X_{2}
\end{aligned} .
$$

The transformation $T_{3}$ may be interpreted as follows. Consider a toxicological experiment in which levels $X_{i}=0$ and $X_{i}=1$ of the toxicants $X_{1}$ and $X_{2}$ denote the presence of the corresponding toxicant at two different levels, namely, $X_{i}=0$ denotes a low dose of $X_{i}$ and $X_{i}=1$ its high dose. Let us suppose that doses $X_{1}=0$ and $X_{2}=0$ are commensurate relative to some effect, as well $X_{1}=1$ and $X_{2}=1$. For example, doses $X_{1}=0$ and $X_{2}=0$ are iso-effective, and $X_{1}=1$ and $X_{2}=1$ as well. This makes it possible to surmise that the combined action type of the toxicants $X_{1}$ and $X_{2}$ at the levels $X_{1}=0$ and $X_{2}=0$, and $X_{1}=1$ and $X_{2}=1$ should be the same, only differing in its power. On the contrary, combined action at the levels $X_{1}=0$ and $X_{2}=1$ may be absolutely different, and at the levels $X_{1}=1$ and $X_{2}=0$ as well. For instance, the dose $X_{1}=1$ may have a much stronger influence than the dose $X_{2}=0$. Thus, the combined action of $X_{1}=1$ and $X_{2}=0$ may be really onefactor one. In such an experiment, the transformation $T_{3}$ preserves the type of the joint action of the toxicants $X_{1}$ and $X_{2}$.

We assume, as previously, that transformation $T_{1}$ preserves the joint action type. Then the group of symmetries of the experiment is generated by the transformations $T_{1}$ and $T_{3}$ being extended over the space of states $\mathbf{B}\left(X_{1}, X_{2}\right)$ according to (5). 
Theorem 6. 1. The group generated by the automorphisms $T_{1}$ and $T_{3}$ is isomorphic to the dihedral group $D_{4}$ (also called the Klein four-group).

2. The $D_{4}$ action on the Boolean algebra $\mathbf{B}\left(X_{1}, X_{2}\right)$ generates the following orbits

$$
\begin{aligned}
& \{0\}, \quad\{1\}, \quad\left\{X_{1}, \bar{X}_{1}, X_{2}, \bar{X}_{2}\right\},\left\{X_{1} \vee X_{2}, \bar{X}_{1} \vee \bar{X}_{2}\right\}, \\
& \left\{\bar{X}_{1} \vee X_{2}, X_{1} \vee \bar{X}_{2}\right\},\left\{X_{1} X_{2}, \bar{X}_{1} \bar{X}_{2}\right\},\left\{\bar{X}_{1} X_{2}, X_{1} \bar{X}_{2}\right\}, \\
& \left\{X_{1} X_{2} \vee \bar{X}_{1} \bar{X}_{2}\right\},\left\{\bar{X}_{1} X_{2} \vee X_{1} \bar{X}_{2}\right\}
\end{aligned}
$$

This partition, in other words, is the classification of the joint action in the binary experiment with the $D_{4}$ group of symmetries.

Proof. The proof can be obtained just the same way as for dihedral group $D_{8}$. The dihedral group $D_{4}$ can be represented by the following relations

$$
D_{4}=\left\{e, T_{1}, T_{3}, T_{1} T_{3}=T_{3} T_{1}\right\}=\left\langle a, b \mid a^{2}=b^{2}=e, b a=a^{-1} b\right\rangle .
$$

Herein, the first equality lists all the elements of $D_{4}$, and the second one represents the group $D_{4}$ as the generators and relations (for references see [7] and [11]).

Alternatively, the Klein-four group $D_{4}$ is isomorphic to the direct product of the two cyclic groups of the second order: $D_{4}=C_{2} \times C_{2}$.

As we can see, the orbits from the previous case (6) are split into sets that are invariant under the action of the group $D_{4}$. One can interpret these orbits similarly to the case of the $D_{8}$-symmetries. It should be noticed that for the $D_{4}$-symmetries the Boolean functions $X_{1} \vee X_{2}$ and $\bar{X}_{1} \vee X_{2}$ represent different combined action types. This is held for other matching pairs of Boolean functions as well.

Having the toxicological experiment in mind as a prototype of the $D_{4}$ symmetries may help to understand this classification. For instance, the conjunction $X_{1} X_{2}$ may exhibit the significant synergism or antagonism, whereas $\bar{X}_{1} X_{2}$ may only represent the influence of $X_{2}$.

It is worth recognizing here that both a classification of the combined action and the notion of synergism are dependent on the presented symmetries of the experiment, i.e., which group of transformations is correct in the case under consideration. For example, in the sufficient causes with the $D_{8}$ group of symmetries the conjunctions $X_{i}^{\alpha} X_{j}^{\beta}$ are considered as exhibiting synergism or antagonism. This viewpoint was argued in [16], [17], [28] and elaborated in [31], [33], [36]. In the experiment with the $D_{4}$-symmetries (e.g., a toxicological experiment with iso-effective levels of the factors, see above) the conjunctions $X_{1} X_{2}$ and $\bar{X}_{1} X_{2}$ have different interaction types. 


\section{Discussion}

In a rigorous formalization of the binary sufficient causes, the theory of Boolean algebras plays a fundamental role. This is to be expected based on the structure of a binary experiment. On the other hand, the importance of experimental symmetries seems to be underestimated. The seminal article [10] does not have further development, though it has been published a long time ago. The natural extension was made in [33] by addition of the transposition invariance. The case of invariance considered in [10] is transparent for the categorized factors as a coding of their levels are arbitrary and only intended for their distinction. Change in reference categories may be incorrect when the levels of the factor are quantitative, possibly on a relative scale. It is typical that the factor's levels distinguish the doses of toxicological exposure in toxicological experiments. Nevertheless, interchangeability of the reference categories may be considered within the toxicological context as well. It may be valid when all possible combinations of levels $X_{1}=i$ and $X_{2}=j$ have the same type of the combined action. This means that levels $X_{1}=0$ and $X_{1}=1$ have the same type of joint action with levels $X_{2}=0$ and $X_{2}=1$. It may be interpreted as the closeness of the levels $X_{1}=0$ and $X_{1}=1$ relative to the considered effect and levels of $X_{2}$.

The presence of certain symmetries in an experiment should be drawn from specific features of the experiment, not from a theoretical model. An experimenter assumes that some transformations preserve automorphisms of the Boolean algebra of states of the experiment. These transformations generate the group of symmetries in the algebraic sense ([7] and [11]). Hence, one can say about the symmetries of a particular group. For instance, classical epidemiological transformations [10] and [33] generate dihedral symmetries $D_{8}$; while in the toxicological transformations (see above) the dihedral group $D_{4}$, or Klein four-group arises.

Generally, one can take a symmetry group that acts on the space of states of an experiment instead of it being generated by some transformations. Since the group of automorphisms of Boolean algebra $\mathbf{B}\left(X_{1}, \ldots, X_{n}\right)$ is the permutation group $S_{n}$ ([8] or [13]), all types of the combined action in a binary experiment with $n$ factors may be obtained through the calculations of the orbits of action of a corresponding subgroup of $S_{n}$. It is reasonable to account only for those subgroups which contain transposition $T_{1}$. For instance, in a binary experiment with two factors such subgroups are $D_{8}, D_{4}$, symmetric group $S_{3}$, and symmetric group $S_{4}$.

It is easy to show that the orbits of the action of group $S_{4}$ are presented by 
the following classes:

$$
\begin{aligned}
& \{0\}, \quad\{1\}, \quad\left\{X_{1}, \bar{X}_{1}, X_{2}, \bar{X}_{2}, X_{1} X_{2} \vee \bar{X}_{1} \bar{X}_{2}, \bar{X}_{1} X_{2} \vee X_{1} \bar{X}_{2}\right\} \\
& \left\{X_{1} X_{2}, \bar{X}_{1} X_{2}, X_{1} \bar{X}_{2}, \bar{X}_{1} \bar{X}_{2}\right\} \\
& \left\{X_{1} \vee X_{2}, \bar{X}_{1} \vee X_{2}, X_{1} \vee \bar{X}_{2}, \bar{X}_{1} \vee \bar{X}_{2}\right\}
\end{aligned}
$$

It is interesting to note that the responses $X_{1} X_{2} \vee \bar{X}_{1} \bar{X}_{2}$ and $\bar{X}_{1} X_{2} \vee X_{1} \bar{X}_{2}$ form a separate class together with the one-factor responses $X_{1}, \bar{X}_{1}, X_{2}, \bar{X}_{2}$. For $D_{8}$ symmetries, functions $X_{1} X_{2} \vee \bar{X}_{1} \bar{X}_{2}$ and $\bar{X}_{1} X_{2} \vee X_{1} \bar{X}_{2}$ have had a specific type of combined action and are included usually in the synergistic type [31], [33], [35]. For $D_{4}$ symmetries, these functions have different types of the joint action, both are not of the one-factor or conjunction types. For more complicated $S_{4}$ symmetries, the responses $X_{1} X_{2} \vee \bar{X}_{1} \bar{X}_{2}$ and $\bar{X}_{1} X_{2} \vee X_{1} \bar{X}_{2}$ are of the one-factor type.

For the $S_{3}$ symmetries types of interaction are presented by the following sets:

$$
\begin{aligned}
& \{0\}, \quad\{1\}, \quad\left\{X_{1}, X_{2}, X_{1} X_{2} \vee \bar{X}_{1} X_{2}\right\}, \quad\left\{\bar{X}_{1}, \bar{X}_{2}, X_{1} X_{2} \vee \bar{X}_{1} \bar{X}_{2}\right\}, \\
& \left\{X_{1} X_{2}, \bar{X}_{1} X_{2}, X_{1} \bar{X}_{2}\right\}, \quad\left\{\bar{X}_{1} \bar{X}_{2}\right\}, \quad\left\{X_{1} \vee X_{2}\right\}, \\
& \left\{\bar{X}_{1} \vee X_{2}, X_{1} \vee \bar{X}_{2}, \bar{X}_{1} \vee \bar{X}_{2}\right\}
\end{aligned}
$$

We see again that the responses $X_{1} X_{2} \vee \bar{X}_{1} \bar{X}_{2}$ and $\bar{X}_{1} X_{2} \vee X_{1} \bar{X}_{2}$ have the same type of interaction as corresponding literals, but the different ones, whereas for the $S_{4}$ symmetries they have the the same type with all the literals. In addition, the responses $\bar{X}_{1} \bar{X}_{2}$ and form separate types of the combined action on their own. One can try to find some interpretation to that kind of the interaction types.

This shows that the precise indication of the group of symmetries of the experiment has fundamental importance in the analysis of joint action types.

\section{Appendix. Some Additional Statistical Criteria for Particular Responses}

In compliance with [10], [20], [31], [33] synergism or antagonism is present in the experiment as soon as an individual is presented with a response of types $C_{M}$ or $C_{S}$. For instance, the definition of definite interdependence [33] is given as follows: Suppose that $D$ and two of its causes, $X_{1}$ and $X_{2}$, are binary. We say that there is definite interdependence between the effect of $X_{1}$ and $X_{2}$ on 
$D$ if there exists an individual $\omega$ for whom $D(\omega)$ can be presented as a Boolean function from the classes $C_{M}$ or $C_{S}$.

In order to determine whether an individual with a specific response exists, one can apply statistical criteria. Let us introduce the following notations to formulate the next theorem [33]. By $p_{i j}=P\left(D=1 \mid X_{1}=i, X_{2}=j\right)$, we denote conditional probability that $D=1$ under $X_{1}=i, X_{2}=j$. The important feature of the binary case is that

$$
P\left(D=1 \mid X_{1}=i, X_{2}=j\right)=\mathrm{E}\left(D=1 \mid X_{1}=i, X_{2}=j\right),
$$

where $E$ denotes the expectation. The theorem (the formulation is given assuming no confounding) states that [33]:

Theorem 7. Suppose that $D$ and two of its causes, $X_{1}$ and $X_{2}$, are binary. If we have that $p_{11}-p_{01}-p_{10}>0$, then there is synergism between $X_{1}$ and $X_{2}$. More exactly, it is stated that when the condition of Theorem 1 is met, an individual of either type $X_{1} X_{2} \vee \bar{X}_{1} \bar{X}_{2}$ or type $X_{1} X_{2}$ must be present.

Below, we prove similar theorems for particular types of response. In what follows hereafter, we use notations from the previous theorem.

Theorem 8. If we have that one of the following inequalities holds

(1) $p_{00}+p_{01}+p_{10}+p_{11}<2$;

(2) $2\left(p_{11} \cdot p_{00}-p_{01} \cdot p_{10}\right)-\left(p_{11}-p_{01}-p_{10}+p_{00}\right)<0$;

(3) $2\left(p_{11} \cdot p_{00}-p_{01} \cdot p_{10}\right)-\left(p_{11}-p_{01}-p_{10}+p_{00}\right)>0$;

(4) $p_{11} \cdot p_{00}-p_{01}-p_{10}>0$;

(5) $p_{01} \cdot p_{10}-p_{00}-p_{11}>0$;

(6) $p_{11}-p_{01}-p_{10}-p_{00}>0$;

(7) $p_{01}-p_{11}-p_{10}-p_{00}>0$;

(8) $p_{10}-p_{11}-p_{01}-p_{00}>0$;

(9) $p_{00}-p_{11}-p_{01}-p_{10}>0$

then, there exists an individual of

(1) one of the types $X_{1} X_{2}, \bar{X}_{1} X_{2}, X_{1} \bar{X}_{2}, \bar{X}_{1} \bar{X}_{2}$ or immune type 0;

(2) one of the types $X_{1} X_{2}, \bar{X}_{1} \bar{X}_{2}$ or $X_{1} \vee X_{2}, \bar{X}_{1} \vee \bar{X}_{2}$; 
(3) one of the types $\bar{X}_{1} X_{2}, X_{1} \bar{X}_{2}$ or $\bar{X}_{1} \vee X_{2}, X_{1} \vee \bar{X}_{2}$;

(4) type $X_{1} X_{2} \vee \bar{X}_{1} \bar{X}_{2}$;

(5) type $\bar{X}_{1} X_{2} \vee X_{1} \bar{X}_{2}$;

(6) type $X_{1} X_{2}$;

(7) type $\bar{X}_{1} X_{2}$;

(8) type $X_{1} \bar{X}_{2}$;

(9) type $\bar{X}_{1} \bar{X}_{2}$.

Proof. The proofs are similar, so it is sufficient to consider only one, and the particular features of the others.

(1) Let us introduce a random variable $X$, defined as follows: $X(\omega)=$ $D_{00}(\omega)+D_{01}(\omega)+D_{10}(\omega)+D_{11}(\omega)$, where $D_{i j}(\omega)$ is the value of the response $D$ for an individual $\omega$ under conditions $X_{1}=i, X_{2}=j$. As $D_{i j}(\omega)$ takes on values 0 or 1 the variable $X(\omega)$ takes on $0,1,2,3$, or 4 . If the inequality $X(\omega) \geq 2$ holds for any $\omega$ then $\mathrm{E}(X) \geq 2$, i.e.,

$$
\begin{aligned}
& \mathrm{E}(X)=\mathrm{E}\left(D_{00}(\omega)+D_{01}(\omega)+D_{10}(\omega)+D_{11}(\omega)\right)= \\
& =\mathrm{E}\left(D_{00}(\omega)\right)+\mathrm{E}\left(D_{01}(\omega)\right)+\mathrm{E}\left(D_{10}(\omega)\right)+\mathrm{E}\left(D_{11}(\omega)\right) \geq 2
\end{aligned}
$$

As mentioned above, $\mathrm{E}\left(D_{i j}\right)=p_{i j}$, thus, $p_{00}+p_{01}+p_{10}+p_{11} \geq 2$. Therefore, there exists such an individual $\omega$ that $X(\omega)<2$, i.e., $X(\omega)=0$ or $X(\omega)=1$. In the first case, the individual $\omega$ is immune, and in the second one, for some individual $\omega$ the single value $D_{i j}(\omega)=1$ while other $D_{i^{\prime} j^{\prime}}(\omega)=0$. The latter case means that full response is one of the next $(1,0,0,0),(0,1,0,0),(0,0,1,0)$ or $(0,0,0,1)$. In logical notations, these responses may be presented as follows: $X_{1} X_{2}, \bar{X}_{1} X_{2}, X_{1} \bar{X}_{2}, \bar{X}_{1} \bar{X}_{2}$. This completes the proof of the point (a).

Other statements can be proved in a similar way. In (4) and (5), one should use that $D_{i j}$ and $D_{i^{\prime} j^{\prime}}$ are independent for $i \neq i$ or $j \neq j^{\prime}$.

Remark: Every inequality (1)-(9) may be tested on statistical significance by an appropriate statistical test constructed similarly to those from [33, Appendix 2]. For instance, a test for statistical significance of inequality (1) is constructed by using following observational quantities:

$n_{i j}$ denotes the number of individuals with $X_{1}=i$ and $X_{2}=j$;

$d_{i j}$ denotes the number of individuals with $X_{1}=i, X_{2}=j$ and $D=1$. 
Then test of the null hypothesis $p_{00}+p_{01}+p_{10}+p_{11} \geq 2$ can be constructed by using critical regions of the following form

$$
\frac{2-\left(\frac{d_{00}}{n_{00}}+\frac{d_{01}}{n_{01}}+\frac{d_{10}}{n_{10}}+\frac{d_{11}}{n_{11}}\right)}{\sqrt{\frac{d_{00}\left(n_{00}-d_{00}\right)}{n_{00}^{3}}+\frac{d_{01}\left(n_{01}-d_{01}\right)}{n_{01}^{3}}+\frac{d_{10}\left(n_{10}-d_{10}\right)}{n_{10}^{3}}+\frac{d_{11}\left(n_{11}-d_{11}\right)}{n_{11}^{3}}}}>Z_{1-\alpha}
$$

to fulfill a one-tailed test (where $Z$ is the standard normal distribution).

The next theorem concerns the interactions of the type $X_{1} X_{2} \vee \bar{X}_{1} \bar{X}_{2}$ or $\bar{X}_{1} X_{2} \vee X_{1} \bar{X}_{2}$. These responses are commonly considered as presenting the synergism or antagonism [10] and [33]. As we have seen above, these Boolean functions form the separate class of combined action, and their interpretation depends substantially on the symmetries of the experiment. In some cases, these Boolean functions have the same type of interaction as one-factor functions $X_{i}$. The following theorem shows that Boolean functions $X_{1} X_{2} \vee \bar{X}_{1} \bar{X}_{2}$ and $\bar{X}_{1} X_{2} \vee X_{1} \bar{X}_{2}$ are present in an almost arbitrary binary experiment. This conclusion casts a doubt on these responses to be considered as synergistic. Rather they have a particular type of interaction that is quite often present in binary experiments.

Theorem 9. If the inequality $p_{00} \cdot p_{11}+p_{01} \cdot p_{10}>0$ holds, then there exists an individual $\omega$ of type $X_{1} X_{2} \vee \bar{X}_{1} \bar{X}_{2}$ or $\bar{X}_{1} X_{2} \vee X_{1} \bar{X}_{2}$, or a dummy one (having a response of 1 in every combination $X_{1}=i, X_{2}=j$ ).

Proof. A random variable $X(\omega)=D_{00}(\omega) \cdot D_{01}(\omega)+D_{10}(\omega) \cdot D_{11}(\omega)$ takes on values 0,1 , and 2 . It is easy to see that $X(\omega) \neq 0$, if the equality $p_{00} \cdot p_{11}+p_{01} \cdot p_{10}>0$ holds. Then either $X(\omega)=1$ or $X(\omega)=2$ is valid. In the first case, an individual of either type $X_{1} X_{2} \vee \bar{X}_{1} \bar{X}_{2}$ or $\bar{X}_{1} X_{2} \vee X_{1} \bar{X}_{2}$ must be present. In the second case, there exists a dummy individual $\left(D_{i j}(\omega)=1\right.$ for $i, j=0,1)$.

There is another reason do not consider the Boolean functions $X_{1} X_{2} \vee \bar{X}_{1} \bar{X}_{2}$ and $\bar{X}_{1} X_{2} \vee X_{1} \bar{X}_{2}$ to be synergistic. Any Boolean function can be considered as a polynomial on the corresponding Boolean ring. The polynomial representation of a Boolean function is often called its algebraic normal form or representation by Zhegalkin polynomials. It is conventional to introduce an interaction term in a model by multiplication of the independent variables. This takes into account nonlinear interaction effects by the interaction term in a linear model. The classification of interaction types considered in the article doesn't depend on the representation of the Boolean function. We might start from Zhegalkin's 
representation as well. Then we would obtain that the responses $X_{1} X_{2} \vee \bar{X}_{1} \bar{X}_{2}$ and $\bar{X}_{1} X_{2} \vee X_{1} \bar{X}_{2}$ have simple linear form:

$$
X_{1} X_{2} \vee \bar{X}_{1} \bar{X}_{2}=1+X_{1}+X_{2}, \quad \bar{X}_{1} X_{2} \vee X_{1} \bar{X}_{2}=X_{1}+X_{2}
$$

Thus, we should consider them as representing no interaction. This makes more understandable that is why these responses may fall into the same interaction classes with literals - the simplest non-trivial linear functions.

\section{Acknowledgements}

This research was supported by Russian Fund for Fundamental Researches (grant \# 12-01-00218).

\section{References}

[1] M. Aickin. Causal Analysis in Biomedicine and Epidemiology, M. Dekker Inc. NY (2002).

[2] R. Allard, J.-F. Boivin. Measures of effect based on the sufficient causes model. 1. Risks and rates of disease associated with a single causative agent. Epidemiology, 4, 1 (1993), 37-42.

[3] Atlas of Finite Groups: Ten Years On, R.T. Curtis and R.A. Wilson (Eds). Cambridge University Press (1998).

[4] S.R. Blackburn, P.M. Neumann, G. Venkataraman. Enumeration of Finite Groups, Cambridge University Press (2007).

[5] R.T. Conway, H. Curtis, S.P. Norton, R.A. Parker, R.A. Wilson. Atlas of Finite Groups, Clarendon Press, Oxford (1985).

[6] R. Cori, D. Lascar. Mathematical Logic. Part I: Propositional calculus, Boolean algebras, Predicate calculus, Oxford University Press (2002).

[7] D.S. Dummit, R.M. Foote. Abstract Algebra, 3rd ed. Wiley and Sons. Hoboken (2004).

[8] S. Givant, P. Halmos. Introduction to Boolean Algebras, Springer, NY (1989). 
[9] S. Greenland, B. Brumback. An overview of relations among causal modelling methods. Int. J. of Epidemiology, 31, 5 (2002), 1030-1037. doi:10.1093/ije/31.5.1030.

[10] S. Greenland, C. Poole. Invariants and noninvariants in the concept of independent effects. Scand. J. Work. Env. Health, 14, 1 (1988), 125-129. doi: 10.5271/sjweh.1945.

[11] P.A. Grille. Abstract Algebra, Graduate Texts in Mathematics. Springer, NY (2007).

[12] GAP Groups and Algorithms. Programming System for Computational Discrete Algebra. http://www.gap-system.org/, 2014.

[13] Handbook Of Boolean Algebras, V. 1-3, J.D. Monk and R. Bonnet, (Eds), Elsevier Science Publishers (1989).

[14] P.W. Holland. Statistics and causal inference. J. Am. Stat. Assoc., 81 (1986), 945-960.

[15] M.J. Khoury, W.D. Flanders, S. Greenland, M.J. Adams. On the measurement of susceptibility in epidemiologic studies. Am. J. Epidemiology, 129, 1 (1989), 183-190.

[16] J.L. Mackie. On causes and conditions. Am. Philos. Quart., 2 (1965), 245255.

[17] J.L. Mackie. The Cement of the Universe: A Study of Causation, Oxford University Press (1974).

[18] B. MacMahon, T.E. Pugh. Causes and entities of decease. In Preventive Medicine, Eds Clark D.W., MacMahon B. Little Brown, Boston (1967).

[19] O.S. Miettinen. Proportion of disease caused or prevented by a given exposure, trait or intervention. Am. J. Epidemiology, 99, 5 (1974), 325-332.

[20] O.S. Miettinen. Causal and preventive interdependence. elementary principles. Scand. J. Work. Env. Health, 8, 3 (1982), 159-168. doi:10.5271/sjweh.2479.

[21] S. Morgan, C. Winship. Counterfactuals and causal inference: Methods and principles for social research. Cambridge University Press (2007). 
[22] V.G. Panov. Some additional sufficient conditions of synergism in the sufficient causes theory. Proceed. TOGU, 1, 1 (2010), 66-69. (In Russian).

[23] V.G. Panov. To the problem of identification of an interaction type of two binary factors in the sufficient causes approach. V Int. Conf. System Analysis in Medicine, (2011), 19-22. (In Russian).

[24] V.G. Panov, J.V. Nagrebetskaya. To the notion of synergism for studies with binary factors. IV Int. Conf. System Analysis in Medicine, Blagoveshchensk. Informatics and Control Systems, 2, 24 (2010), 22-25.

[25] V.G. Panov, J.V. Nagrebetskaya. An algebraic interpretation of two factor binary sufficient causes theory. Proceed. SPIIRAN, 26 (2013), 227-246. (In Russian).

[26] J. Pearl. Causality: Models and Reasoning and Inference, Cambridge University Press (2000).

[27] Wolfram Research. Wolfram Mathworld. Wolfram Research, 2014.

[28] K. Rothman. Causes. Am. J. Epidemiology, 104, 6 (1976), 587-592.

[29] S. Schwartz, N.M. Gatto, U.B. Campbell. Extending the sufficient component cause model to describe the Stable Unit Treatment Value Assumption (SUTVA). EP+I, 3;9:3 (2012). doi: 10.1186/1742-5573-9-3.

[30] The Group Properties Wiki. Symmetric group. http://groupprops.subwiki.org/wiki/Symmetric_group.

[31] T. J. VanderWeele. Sufficient cause interactions and statistical interactions. Epidemiology, 2009, 20, 1 (2009), 6-13. doi: 10.1097/EDE.0b013e31818f69e7

[32] T.J. VanderWeele. The sufficient cause framework in statistics, philosophy and the biomedical and social sciences. In Causality: Statistical Perspectives and Applications, Eds. Berzuini C., Dawid P., Bernardinelli L., Wiley \& Sons, 180-193, (2012).

[33] T.J. VanderWeele, Robins. J.M. The Identification of Synergism in the Sufficient-Component-Cause Framework. Epidemiology, 18, 3 (2007), 329339. doi: 10.1097/01.ede.0000260218.66432.88. 
[34] T.J. VanderWeele, T.S. Richardson. General theory for interactions in sufficient cause models with dichotomous exposures. Ann. Statist., 40, 4 (2012), 2128-2161. doi: 10.1214/12-AOS1019.

[35] T.J. VanderWeele, J.M. Robins. Empirical and counterfactual conditions for sufficient cause interactions. Biometrika, 95, 1 (2008), 49-61. doi: 10.1093/biomet/asm090.

[36] T.J. VanderWeele, J.M. Robins. Theory of sufficient cause interaction, COBRA Preprint Series, article 13 (2009). http://biostats.bepress.com/cobra/ps/art13.

[37] S. Vansteelandt, T.J. VanderWeele, J.M. Robins. Semiparametric tests for sufficient cause interaction. J. R. Stat. Soc., 74, 2 (2012), 223-244. doi: 10.1111/j.1467-9868.2011.01011.x. 
